scores across life with objective biomarkers of cardio-metabolic health.

Methods Analyses were undertaken among 4820 civil servants aged 59-83 years, within the prospective Whitehall II study, who answered the life-grid AUDIT-C inventory during the 2011-2012 survey and provided biological measurements (264 non-drinkers were excluded). Lifetime hazardous drinking groups were defined using a threshold of $\geq 5$, at each decade of life from age 16 to $80+$. These groups were as follows; never hazardous-drinker (reference), former hazardous-drinker 1 (before age 50), former hazardous-drinker 2 (after age 50), current hazardous-drinker (past hazardous-drinker sporadically), stable hazardous-drinker (hazardous-drinker in every decade). Similar groups were created for lifetime binge-drinking categories; never/former/current/stable binge-drinker (AUDIT-3 $\geq 2$ ). Fully-adjusted linear regression was carried out on cardio-metabolic biomarkers including: waist circumference (WC, measured in $\mathrm{cm}$ ), body mass index $\left(\mathrm{BMI}, \mathrm{kg} / \mathrm{m}^{2}\right)$, total cholesterol (TC, mmol/L), systolic (SBP, $\mathrm{mmHg}$ ) and diastolic (DBP, $\mathrm{mmHg}$ ) blood pressure, gamma-glutamyl transferase (GGT), fatty-liver index scores (FLI) and lifetime hazardous/binge drinking as exposure, using STATA15. Covariates included sex, age, socio-economic position, ethnicity, smoking status, physical activity, BMI and fruit and vegetable consumption.

Results Over half of the sample had been a hazardous-drinker at some point; Current hazardous-drinkers (21\%), former hazardous-drinkers $_{1}$ (<age 50) (19\%), former hazardous-drinkers 2 ( $\geq$ age 50) (11\%) stable hazardous-drinker (5\%). After adjusting for co-variates, hazardous-drinkers had a larger WC than never hazardous-drinkers (former hazardous-drinkers ${ }_{1} \quad(\beta=1.17$ [95\% CI 0.25, 2.08]); (former hazardous-drinkers 2 (1.88 [95\% CI $0.77,2.98])$; current hazardous-drinkers (2.44 [1.55, 3.34]) and stable hazardous-drinkers (3.85 [2.23, 5.47])). A similar linear association along more current and frequent hazardousdrinking was also found with BMI. Current hazardous-drinkers had higher SBP $(2.44[1.19,3.68]), \log$ (GGT) (22.64 [18.27,27.02]) and FLI scores (4.05 [2.92, 5.18]) than never hazardous-drinkers, and so did stable hazardous-drinkers (sbp (2.78 [0.53, 5.04]), $\log (\mathrm{GGT}) \quad(17.94$ [10.12, 25.75]), FLI (3.76, [1.75, 5.77])). Similar associations with waist, sbp, GGT, and FLI outcomes were found for lifetime bingedrinkers.

Conclusion Hazardous-drinking is common among older adults and may increase cardio-metabolic risk factors, this may be compounded by persistent hazardous-drinking across life. Population reductions in hazardous-drinking is likely to have immediate improvements in elderly, but also long lasting improvements with early intervention in the life course, particularly with weight gain. Future analyses will assess risk of lifetime hazardous-drinking and cardiovascular events and mortality.

\section{OP24 SUBCLINICAL CARDIOVASCULAR DISEASE AND FRAILTY AMONG BRITISH OLDER MEN WITHOUT A DIAGNOSIS OF CARDIOVASCULAR DISEASE: A POPULATION-BASED STUDY}

${ }^{1} \mathrm{M}$ Patel*, ${ }^{*} \mathrm{AO}$ Papacosta, ${ }^{1} \mathrm{LT}$ Lennon, ${ }^{2} \mathrm{EA}$ Ellins, ${ }^{2} \mathrm{~J}$ Halcox, ${ }^{3} \mathrm{PH}$ Whincup, ${ }^{4}$ SE Ramsay, ${ }^{1} \mathrm{SG}$ Wannamethee. 'Department of Primary Care and Population Health, University College London, London, UK; ${ }^{2}$ Institute of Life Sciences, Swansea University, Swansea, UK; ${ }^{3}$ Population Health Research Institute, St George's University of London, London, UK; ${ }^{4}$ Institute of Health and Society, Newcastle University, Newcastle upon Tyne, UK
Background Frailty is associated with incident cardiovascular disease (CVD). However, its association with subclinical CVD, which precedes the onset of a CVD event, remains uncertain. Non-invasive vascular markers provide valuable proxy indicators of CVD risk, permitting investigation of risk burden and the development of subclinical CVD. N-terminal pro brain natriuretic peptide (NT-proBNP) and cardiac troponin $\mathrm{T}$ (cTNT) are markers of cardiac injury and are strong risk predictors of CVD events such as heart failure and myocardial infarction respectively. Our aim was to examine the association of subclinical cardiovascular markers with frailty in men without a diagnosis of CVD.

Methods In 2010-2012, 1622 surviving men aged 71-92 years were examined and completed a questionnaire as part of a cross-sectional study from a cohort of men from 24 British towns initially recruited in 1978-1980. Using the Fried phenotype, frailty was defined by the presence of $\geq 3$ of the following components: unintentional weight loss, low grip strength, low physical activity, slow walking pace and exhaustion. Carotid intima media thickness (CIMT) and carotid distensibility coefficient (DC) were measured using ultrasound. A Vicorder device was used to measure pulse wave velocity (PWV) and ankle brachial pressure index (ABPI). Fasting blood samples were analysed for NT-proBNP and cTNT. Multivariable logistic regression was used for analyses.

Results Three hundred and three participants (19\%) were frail and $876(54 \%)$ were pre-frail. In men without CVD, frail individuals were significantly more likely to be older, physically inactive, have a smoking history and co-morbidities such as peripheral vascular disease and diabetes compared to nonfrail individuals $(\mathrm{p}<0.05$ for all associations). Multivariable analyses of baseline demographics and diabetes showed that frailty was positively and significantly associated with high NT-proBNP (odds ratio $[\mathrm{OR}]=3.48 ; 95 \%$ confidence interval $[\mathrm{CI}=2.13-5.67)$, high cTNT $(\mathrm{OR}=3.33 ; 95 \% \mathrm{CI} 2.03$ to 5.45) and low DC (reflecting greater arterial stiffness; $\mathrm{OR}=1.79$; $95 \% \mathrm{CI} 1.11$ to 2.88 ). Some subclinical vascular markers (ABPI $<0.9$ or $>1.2$, PWV and CIMT) were not associated with frailty $(\mathrm{p}>0.05)$. Pre-frailty was also associated with raised cardiac markers $(\mathrm{p}<0.05$ for all associations).

Conclusion Frailty is strongly associated with subclinical CVD markers (NT-proBNP, cTNT and DC), even in the absence of prevalent CVD. Further research is needed to determine whether this association is causal and to understand the underlying mechanisms. Our study findings support the need for screening older individuals with frailty, with the objective of identifying those with an underlying increased risk of CVD and its adverse outcomes.

\section{OP25 SOCIOECONOMIC FACTORS ASSOCIATED WITH FRAILTY: RESULTS FROM TWO STUDIES OF OLDER BRITISH POPULATIONS}

${ }^{1}$ SE Ramsay*, ${ }^{2} \mathrm{E}$ Papachristou, ${ }^{2} \mathrm{AO}$ Papacosta, ${ }^{2} \mathrm{LT}$ Lennon, ${ }^{3} \mathrm{PH}$ Whincup, ${ }^{2} \mathrm{SG}$ Wannamethee. ${ }^{1}$ Institute of Health and Society, Newcastle University, Newcastle upon Tyne, UK; ${ }^{2}$ Institute of Epidemiology and Health Care, University College London, London, UK; ${ }^{3}$ Population Health Research Institute, St George's University of London, London, UK

\subsection{6/jech-2018-SSMabstracts.25}

Background Frailty is a state of increased vulnerability to stressors in older age, which increases risks of disability, falls and mortality. Prevalence of frailty is very high in older populations. The extent to which socioeconomic factors are 
associated with frailty is less well studied. We investigated the extent to which socioeconomic factors at individual and area level are associated with frailty in two studies of older populations in the UK.

Methods Data are from two studies of older populations: the British Regional Heart Study (BRHS) comprised a socially representative sample of men $(n=1622)$ from 24 British towns aged 71-92 years in 2010-12; the English Longitudinal Study of Ageing (ELSA) comprised a representative sample of older men and women $(n=5344)$ aged $\geq 60$ years in 2004 from England. Using the Fried phenotype, frailty was defined by the presence of $\geq 3$ of the following components: unintentional weight loss, low grip strength, low physical activity, slow walking pace and exhaustion. Socioeconomic measures included occupational social class and area-level deprivation was based on the Index of Multiple Deprivation (IMD). Logistic regressions models were used.

Results Prevalence of frailty was $19 \%$ and $9 \%$ in the BRHS and ELSA populations respectively. In the BRHS sample, the risk of frailty increased from the highest (social class I) to lowest social class V; age-adjusted odds ratio was 1.18 (95\% confidence interval $(\mathrm{CI})=1.07-1.31)$ for each category from social class I to $\mathrm{V}$, which remained significant on adjustment for smoking, history of cardiovascular disease (CVD) or diabetes, body mass index (BMI) and alcohol consumption. The risk of frailty also increased from the least (quintile 1) to most deprived IMD quintile (quintile 5); OR per quintile $=1.19 \quad(95 \%$ CI 1.08 to 1.30$)$. This increased risk remained significant on further adjustment for covariates. Similarly, in the ELSA population of older men and women, frailty risk was greater in lower social classes $(\mathrm{OR}=1.21 \quad(95 \% \mathrm{CI}$ 1.16 to 1.27$)$ for each group from highest to lowest social classes. Frailty risk was greater also in deprived quintiles $(\mathrm{OR}=1.35,95 \% \mathrm{CI} 1.28$ to 1.43 , for every increase in quintile of deprivation). These associations remained significant on adjustment for covariates.

Conclusion Adverse socioeconomic factors are associated with risk of frailty in older populations. These associations were independent of lifestyle factors and comorbidities, and were observed both for individual and area-level socioeconomic factors. Socioeconomic factors are potentially important in reducing the burden of frailty in older people. These findings merit further investigation prospectively.

\section{Thursday 6 September 2018}

\section{Ageing and mental health}

\section{OP26 TEMPORAL TRENDS IN MULTI-MORBIDITY AND HOW IT IMPACTS EMPLOYMENT AMONG OLDER ADULTS IN CANADA AND ENGLAND: UNDERSTANDING GENERATIONAL AND SOCIAL INEQUALITIES}

${ }^{1} \mathrm{~L}$ Bentley*, ${ }^{2} \mathrm{Q}$ Liao, ${ }^{1} \mathrm{~B}$ Barr, ${ }^{2} \mathrm{C}$ Mustard. 'Department of Public Health and Policy, University of Liverpool, Liverpool, UK; ${ }^{2}$ Institute for Work and Health, Toronto, Canada

\subsection{6/jech-2018-SSMabstracts.26}

Background In most countries policy makers are seeking to raise the age at which people become eligible for state pensions due to population aging. Aging societies are also characterised by an increase in the number of people are living with chronic conditions and multi-morbidity which can affect employment later in life. This study examines the prevalence of multi-morbidity by age, birth-cohort, and socioeconomic status before assessing the impact that this has on employment status for workers approaching retirement age. It presents a comparative analysis of the UK and Canada and discusses the different policies that each have adopted regarding older workers with chronic conditions.

Methods We estimate the prevalence of multi-morbidity (composed of 6 broad categories of disease) for those aged between 50 and 64, by sex, educational level, and by two birth cohorts: the silent generation (pre-1946) and the baby boomers (1946-1964), using the Health Survey for England and the Canadian Community Health Survey. The prevalence of each condition and the changes in specific comorbidities between the generations were estimated. Finally, the association between multi-morbidity on the employment status (in employment or not) is assessed. Each of the analyses uses logistic regression models.

Results Prevalence of multi-morbidities has increased between generations, particularly for the lower educated in England and higher educated groups in Canada. As the number of conditions increased the probability of being in employment decreased. While employment rates are similar for people with no-conditions in the UK and Canada there was a more negative impact of having more than one condition in the UK. For example, silent generation women with a low education in the England with $3+$ conditions were much less likely to be employed than women with no health conditions (odds ratio of $0.095(0.071-0.128)$, whilst the effect was lower for low educated silent generation women in Canada 0.318 $(0.235-0.431))$. The consequences of multi-morbidity for employment was found to be similar between generations. The increase in multi-morbidities may have led to reduced employment prospects particularly amongst the less educated groups in England.

Conclusion Many countries are facing the challenge of an aging population. The effect of multi-morbidity on employment for older workers has been understudied even though it presents a set of challenges which risk exacerbating existing social inequalities because they are concentrated among less advantaged groups and associated with reduced employment prospects. The research presented here suggests that different policy approaches might be effective in ameliorating these inequalities.

\section{OP27 PSYCHOLOGICAL FACTORS ASSOCIATED WITH STANDING BALANCE PERFORMANCE AT AGE 69 IN A BRITISH BIRTH COHORT STUDY}

JM Blodgett*, F Harkness, D Kuh, DHJ Davis, R Cooper. MRC Unit for Lifelong Health and Ageing, University College London, London, UK

\subsection{6/jech-2018-SSMabstracts.27}

Background Balance ability from midlife has been consistently shown to predict future health outcomes including mortality, morbidity and falls risk. Evidence has identified socioeconomic, behavioural and anthropometric contributors to balance, however there is little evidence on the role of psychological factors. We aimed to examine the associations between five psychological factors - stressful life events, symptoms of anxiety and depression, wellbeing, mastery and fear of falling - and standing balance performance at age 69 . 